\title{
UNIVERSAL DIGITAL ENVIRONMENT MODEL FOR COMPUTER INTEGRATED ROAD CONSTRUCTION
}

\author{
Jarosław Jurasz \\ Institut für Maschinenwesen im Baubetrieb (Institute of Construction Management and \\ Machinery), University of Karlsruhe, Am Fasanengarten 76128 Karlsruhe, Germany \\ tel. +49 721 6083884, e-mail: jurasz@imb.uni-karlsruhe.de, \\ http://www.uni-karlsruhe.de/ gm07
}

\begin{abstract}
As the computer supported road construction systems evolve, the issues of compatibility and inter-operability start to play more important role in than the limitations posed by computing resources. The paper discusses modelling methods currently in use and presents a novel, efficient and flexible environment description for on-board systems in this context. The results of its application, conversion methods and data exchange issues are discussed extensively.
\end{abstract}

Keywords: computer integrated road construction, ribbon, digital terrain model, environment model, road product model, on-board computer

\section{INTRODUCTION}

Nowadays the construction machines are increasingly often equipped with the on-board computers, which support the operator and take over the difficult and cumbersome control and documentation chores ([Ci00] [Pe99] [Th01]). Their application is especially promising in civil engineering, where the machines perform repeatable and well-defined tasks [Pe00]. Users wish to participate more actively in the quality control, especially in new contract models, e.g. Build-Own-Operate.

Thanks to advances in positioning technology, on-board communication and following the need of the users, the on-board IT plays increasing role on the modern road construction site.

With the increasing number and extending functionality of support systems in existence, the problems of interoperability and standardisation start to play a major role. Differently equipped machines from several vendors have to co-operate in order to perform the common task. Depending on the kind of machine, the level of provided on-board support may be different, yet common data models are needed in order to enable the co-operation.

This paper presents a universal on-board description of the worksite, developed in the frameworks of CIRC (Computer Integrated Road Construction, http://pro.wanadoo.fr/pges/circ [Ci00] [Pe00]) and Osyris (Open System for Road Information Support, http://www.osyris.org [Os01]) European Projects, also known as a ribbon description. The ribbon description forms an on-board part of Osyris Road Product Model [Os01]. It is focused on road construction site, although presented approach can be applied to other linear civil engineering structures, like railways, canals or tunnels.

We start with the problem statement, then a comparative overview of existing on-board data models is given, followed by the presentation of the ribbon model. Results of its application and conversion methods to other models are discussed. The analysis proceeds with respect to the quality and performance of representation.

\section{PROBLEM STATEMENT}

The IT system for road machine support has to deal with several kinds of objects, for example lines and surfaces describing designed road, curvilinear reference frame, and additional objects like surveyor monuments, bridges or orientation signs. Beside these geometrical entities equipped with physical attributes, non-geometrical entities like machine fleet or work team need to be modelled.

Traditionally, the term Digital Terrain Model (DTM) of GIS origin [F198] has been used to describe the required modelling as a whole, but it is too limited taken into account the diversity of required entities. Therefore in this article we adopt the term Digital Environment Model (DEM), coming from the context of robotics.

Many of the entities mentioned above originate from the process of the road design, where they are usually modelled as concatenation of line segments, clothoids and arcs. In simplified form the design entities can be also given as discrete 2 or $3 \mathrm{D}$ polylines. Some designed process attributes, like a 
material type or an optimal temperature range are often not provided digitally.

In the course of the project execution the designed description is augmented by the trajectory of machine tool, as defined by the positioning and the tool geometry. The process parameters, e.g. the material temperature or the current vibration amplitude can be attached to each position, thus forming the description of the actual road surface, but also of the processes which influenced it.

The geometrical objects mentioned lie mostly along the site axis and can be conveniently represented in the curvilinear co-ordinate system. They exist within elongated and flat realm, specific to road construction. It is also anisotropic in the sense, that characteristic sizes in a curvilinear co-ordinate system differ widely, for example the typical grid size could be $2 \mathrm{~m}$ in longitudinal, $0.5 \mathrm{~m}$ in lateral and 1 $\mathrm{cm}$ in the vertical direction. That is why specialised ways of representing the road objects are needed, as compared to methods applied in all-purpose CAD and CAM systems.

Following Peyret's classification [Pe99], the universal DEM should support the following groups of machines:

a) Surfacing machines (eg. compactors)

b) Profiling machines (eg. pavers, graders)

c) Earth moving and mining machines (eg. bulldozers, wheel loaders)

d) Drilling machines.

On an asphalt pavement worksite, data exchange is required between surfacing and profiling machines, whereas in the case of earthwork site the cooperation between the earth moving and profiling machines is necessary. We do not discuss the drilling machines in this paper. Two operation modes are possible: online exchange through the wireless site network or offline data gathering using mass memory medium. In any case, the DEM should allow for efficient combining and exchanging of data between different machine groups.

\section{STATE OF THE ART}

The DEMs in current use follow one of the two dual paradigms: raster or vector description. Vector description is an entity based view, where objects fill up the space, and raster description is a space oriented view, where each point in space has some properties. Neither model appears to be superior in all tasks.

Taking into account the application area, the models in use can be further classified into the following groups:

* Raster descriptions are simple and effective, but lousy in the sense, that the rasterisation process cannot be inverted. Conversion between different rasters is difficult and the produced image of the achieved work is not intuitive. Raster modelling is commonly used for compaction documentation [Th01].

* Multiple polyline (multi-polyline) representations, also known as spaghetti models [F198], are simplified DTMs as used by many levelling systems. They allow exact tool control, but are often limited to the design description only. The polylines represent longitudinal or cross profiles. Existing implementations often pose limitations, for example the points have to be implicitly paired.

* DTMs based on Triangulated Irregular Networks (TINs) originate from surveying and GIS applications and are focused at representing a static, complex 3D geometry. They are used for earthwork applications. However, the tool trajectory is not readily described by triangles. Moreover, in this geometry-centric paradigm the process parameters are difficult to place. They should be attributed to triangle edges, which are normally not stored as entities.

* The road network databases are complex systems organised around road network topology. They may use a mixture of curvilinear and geographical description and cover wide application areas They are very diverse, depending on national practice. One example of a modern road network model is the German OKSTRA ${ }^{\circledR}(\mathrm{Ob}-$ jektkatalog für Straßen- und Verkehrwesen, http://www.okstra.de).

It can be easily seen that conversion of information between these descriptions can be a cumbersome task, and information loss is inevitable.

\section{RIBBON DESCRIPTION}

The ribbon description is based on the observation that the smooth curves and surfaces of the road can be well approximated by multiple piecewiselinear structures, like polylines and sets of polygons. The ribbons described in this article are basically concatenations of quadrilaterals (see Figure 1.). However, to model triangles and polylines it is necessary to allow the degenerated (double) vertices. The ribbon may have at most 2 degenerate vertices, thus the quadrilaterals may be reduced to triangles or line segments. The points can be modelled by the vertices alone.

Our ribbon can be viewed as a generalisation of a polyline; it is a polyline with width, which is oriented in 3D space. The width is non-negative and may vary along the ribbon. For example, the width is a non-zero constant for ribbon describing the surface 
treated by the machine, variable for road surface ribbon and zero for line ribbons.

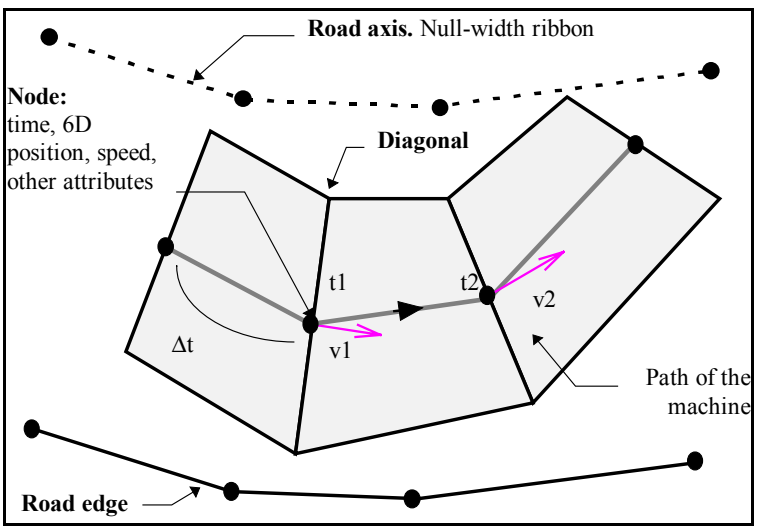

Figure 1. Three sample ribbons

Some classes of continuous ribbons and their basic properties are discussed in a survey by Rosenfeld [Ro86]. Given a curve called "axis" or "spine" and a planar figure called generator, the ribbon can be created by moving the generator along the axis. Our ribbon can be classified as a discrete, generalised, case of Brooks ribbon. Brook's ribbon generator is a line segment making fixed angle with the axis, in our case this is not required.

Bhattacharya and Rosenfeld [Bh95] have studied geometric and topological properties of discrete ribbons, concentrating on triangular and rectangular ones. It has been shown that quadrangular data models have advantages over triangles in the domains of scattered data analysis and elasticity analysis based on finite element methods. Continuous ribbons are used in modelling of DNA structures.

The length of the generator is called ribbon width. The ribbon axis is itself a ribbon of the width zero. The vertices of the ribbon axis are called ribbon nodes. The co-ordinates and the width are the obligatory node parameters. It is optional for the node to have various attributes, for example time or speed.

\section{CONVERSION ALGORITHMS}

\subsection{Ribbonisation}

The incoming data is converted into ribbons in the process called "ribbonisation". Points, polylines and processed areas can be readily inserted into ribbons. Thus, conversion from raster and line representations to the ribbons is straightforward. However, incoming polygons and surfaces have to be quadrangulated prior to inserting into the ribbons.

Ribbonisation of the design geometry is commonly performed only once per session and is therefore not time-critical. However, its quality has great impact on the memory requirements and the time complexity of the further processing, in particular on the visualisation process.

A multi-polyline model, as well as "narrow" polygons, can be effectively converted into ribbons in linear time by Delaunay ribbonisation (see Figure 2.). The Delaunay tessellations are preferred in many applications, as they are known to maximise the minimal angle, minimise the maximum circumcircle and minimise the maximum containing circle [F198].

This algorithm proceeds incrementally along the curvilinear axis, choosing the shortest of three possible diagonals. Triangles can be inserted to improve the renresentation In this wav the skewed data and

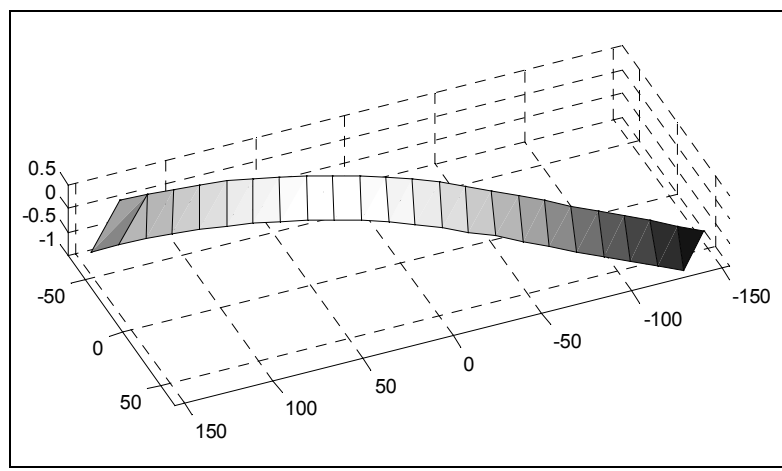

Figure 2. Delaunay ribbonisation of a multipolyline road design

The more complex problem of ribbonisation of TIN DTM is specific to the earth moving application and is covered in greater detail in Section 6.3.

\subsection{Conversion to other representations}

The ribbon description can be converted into raster in real time, for example in the visualisation pipeline. The time complexity is clearly linear. As all the vertices are preserved, the multi-polyline and triangulated DTM can be created easily.

The curvilinear co-ordinates are often required to export the work description to the road in format suitable for network database.

The ribbons can be expressed in the curvilinear, rather than Cartesian co-ordinate system. The case is very important in the practice, as simple curvilinear positioning devices (e.g. odometers, sonic distance meters) are much cheaper and than the absolute ones (e.g. GPS, robotic total stations).

If the ribbons are expressed in the Cartesian coordinate system and the curvilinear reference frame has been ribbonised, it is possible to efficiently convert between the curvilinear and the Cartesian coordinate space using the geometric search and projection primitives. In an incremental case, as for the moving machine, the conversion to curvilinear coordinates can be done in a constant time. 


\section{APPLICATION RESULTS}

\subsection{Surfacing support: compactor}

In the surfacing case the pass map is of particular interest for the operator. It helps to obtain the uniform process result, for example compaction.

The compactor trajectory is described by a selfintersecting ribbon. It is discontinuous due to work breaks, missing positioning data etc. An example of support map based on ribbons, as implemented in CIRCOM system is shown in Figure 3.

The pass map can be effectively calculated in linear time, allowing for a real-time visual feedback (the screen update rate can be as high as $5 \mathrm{~Hz}$ ). The dominant axis has influence on the MMI design, favouring the page-oriented map presentation.

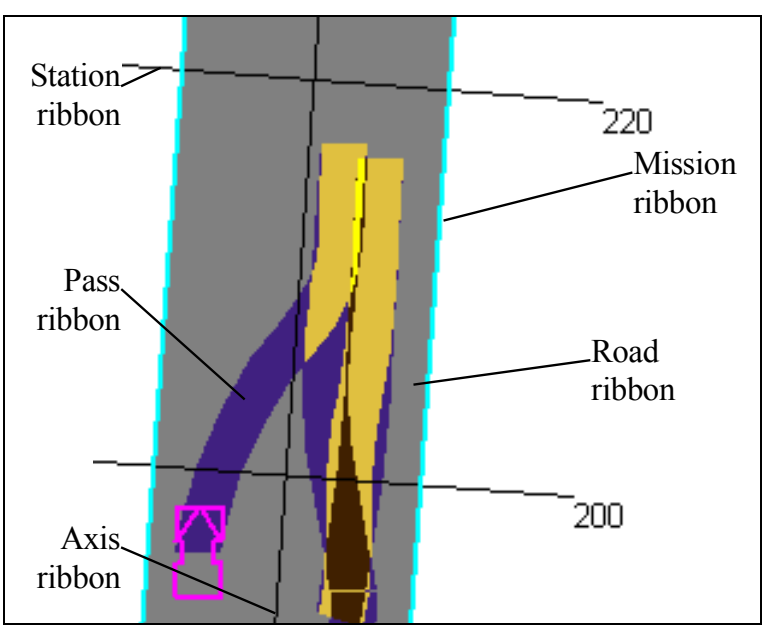

Figure 3. Ribbons visualised in compactor system - CIRCOM MMI

In the multi-machine environment the ribbon node is a natural unit of data exchange algorithm [Ci00]. Information management is greatly simplified by ordering imposed by the ribbon representation.

Moreover, thanks to the uniform environment modelling method, it is possible to include the paver in the process information exchange. Thus the laying information can be used to optimise the compaction process. For the sake of universality, the 3D approach may be used uniformly, also for apparently 2D compactor systems. Cost of storing the additional co-ordinate is compensated by uniform handling.

The ribbon description is permanent; it can be quickly stored in mass memory before the system is switched off and loaded upon start. The contents of DEM can be stored repeatedly, so that in the case of the system failure, the last saved state can be restored.

As all the movements of the machines are stored in ribbons, it is possible to export and extensively analyse the work progress and process information.
Alternatively, it is possible to export the pass map in raster form.

\subsection{Profiling support: paver, grader}

The main task of the on-board system on the profiling machine is the calculation of the designed elevation, given the current position. The MMI (as implemented in CIRPAV system) consists of an overview map, a cross-section view and a trajectory view (see Figure 4.).

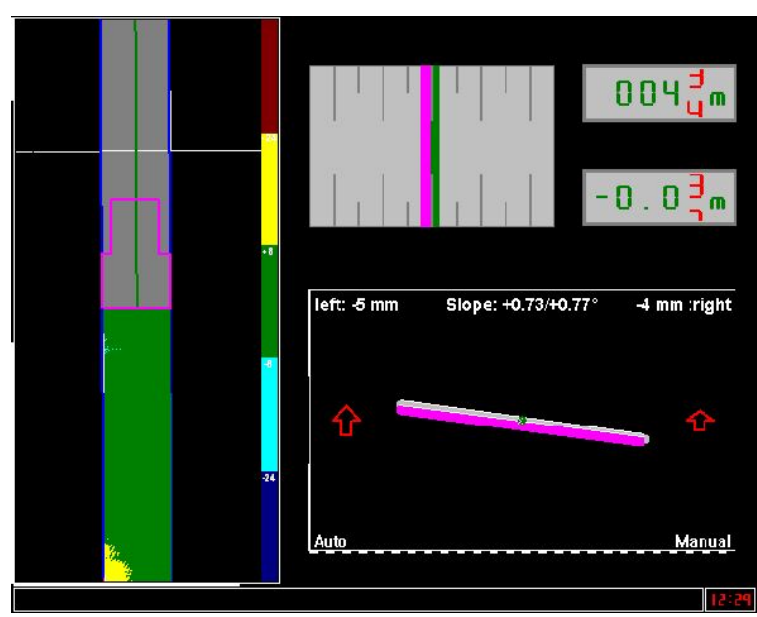

Figure 4. Ribbons in profiling application - CIRPAV MMI

In the $3 \mathrm{D}$ case the principle of the ribbon representation doesn't guarantee, that the quadrilaterals will be planar (as opposed to the triangular representation). This is not a serious problem in road construction applications, as the represented surfaces are reasonably flat by the design and the process nature. Moreover, the following interpolation strategies can be applied to calculate intermediate values of the ribbon attributes:

* linear interpolation along curvilinear axis

* bilinear interpolation within quadrilateral

* triangular (barycentric) interpolation. 1 additional bit is required to store the triangular partitioning of quadrilateral

* stepwise extrapolation - especially suitable for attribute values

The interpolation concerns not only the elevation, but also other process parameters.

To assure robust interpolation in the profiling application it is important to guarantee non-self intersection (NSI, [Bh95]) property of generated ribbons.

The flatness of the ribbon quadrilaterals can be verified upon input. The concept of a set width ${ }^{1}$, well

${ }^{1}$ Given a set of points $\mathrm{P}$, its width is a minimum distance between two parallel planes that limit $\mathrm{P}$ 
known in the computational geometry, can be used to measure the flatness of quadrilateral.

The flatness guarantee is especially important in levelling tasks and should be kept below the required profiling accuracy (the sub-millimetre range in the case of asphalt pavement). Insufficient flatness indicates poor quality of the design data or too low positioning accuracy or frequency.

\subsection{Earthmoving support: bulldozer}

To support the earthmoving process a fullfledged DTM is needed, based on points surveyed beforehand and updated in a real-time. The main task of the on-board system in this case is to monitor the volume transfer and calculate and visualise the discrepancies between the actual and the designed surface.

As a triangle is a ribbon of length 1 (with two overlapping vertices), any triangulated surface with $\mathrm{N}$ triangles can be identity-converted into a concatenation of $\mathrm{N}$ ribbons. However, with two nodes per triangle face, this "naive" representation is not optimal and costs four times more with respect to memory and time compared to the connected ribbon of length $\mathrm{N}$. As mentioned before, finding a good 3D ribbonisation of a DTM based on a TIN is not trivial.

Let's consider a dual graph of triangulation, that is the graph of connections between triangles via common edges. The "naive" representation can be improved by joining the adjacent triangles along the paths in the graph. Moreover, joining of triangles into quadrilaterals may further decrease the number of required nodes. This is possible whenever the entry and exit edges of the quadrilateral are not adjacent ("opposite doors" property).

The related problem of finding optimal triangle strips for fast visualisation of triangulated surfaces has been widely studied by the computational geometry community. The optimal solution requires visiting all triangles along the Hamiltionian path ${ }^{2}$. The problem of finding it is related to the travelling salesperson problem, and thus NP-hard [Ar94]. The additional condition posed by "opposite doors" rule requires the triangulation to be sequential, that is the turns in the path need to alternate left-right. The sequential, Hamiltionian triangulation can be used to reduce the number of required nodes, memory and processing time by factor of four and produce the optimal ribbon

However, solving the 3D ribbonisation problem optimally is not practical. In the practically important case of the regular and almost-regular grids the $3 \mathrm{D}$ ribbonisation is particularly easy. In the case of irregular grid, fast heuristic methods [Xi99] are

\footnotetext{
${ }^{2}$ Hamiltonian cycle/path in undirected graph is a simple cycle/path connecting all vertices. Such a graph is called hamiltonian
}

known to find sub-optimal sequential strips in linear time. The achieved reduction amounts to 2.7 in the practical case shown in Figure 5.

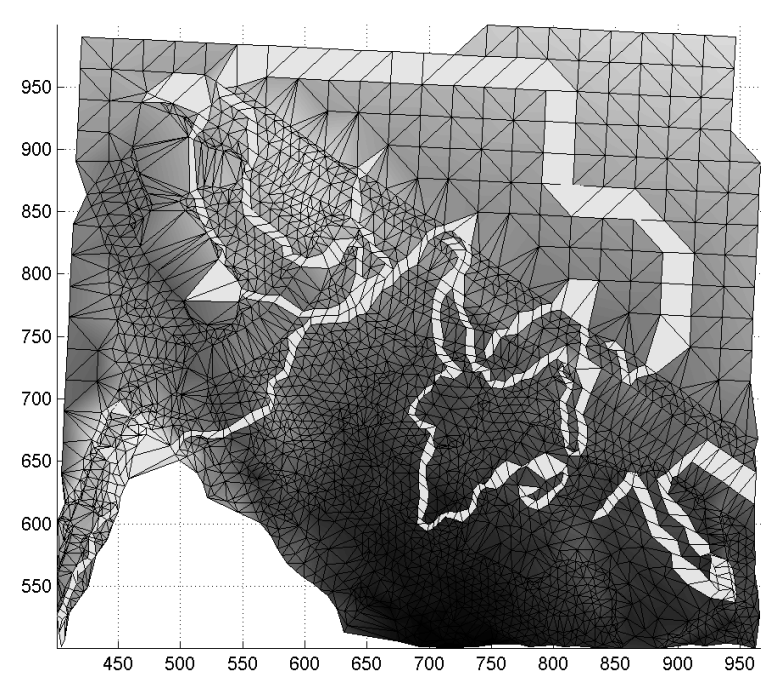

Figure 5. 5 longest ribbons (50..100 nodes each) in the DTM with $5 \mathrm{k}$ triangles and 381 ribbons. The overall number of nodes was reduced from $10 \mathrm{k}$ to $4 \mathrm{k}$ using fast heuristic described in [Xi99].

After a good ribbonisation of an initial state has been found, it needs to be effectively updated in the real time, as the work proceeds. A further research is needed to verify if it is better to repeatedly modify the existing ribbons so as to ensure the NSI property (similar to the profiling system), or to collect the machine paths and create the terrain map cumulatively in the rasterisation process (as in the surfacing system).

\section{CONCLUSIONS}

The main features of the presented ribbon data model are:

* Universality: all the relevant objects on the work site are represented in uniform and coherent way. The possibility of efficient conversion to and from other representations in current use is assured.

* Object paradigm: the physical attributes are compactly stored in the database together with the geometrical objects they apply to.

* Support for the online (site network) and offline data exchange

* Exact and fast visualisation.

* Safe permanence: the database is robust and remains safe in the case of power or system failure. 
The ribbon data model offers the flexibility and the performance required for the universal on-board representation of the environment in road construction. It can be applied for all three required machine groups. As opposed to the other data models in use, the ribbon model can accommodate both complex geometry and physical process attributes, offers machine independence and enables inter-machine co-operation.

\section{Acknowledgements}

The work described in the paper was carried out in the frameworks of the following European Projects:

* CIRC: Computer Integrated Road Construction project (Brite-EuRam III No. BE-96-3039) 1997-2000

* OSYRIS: Open System for Road Information Support (Growth No. GRD1-1999-10815), 2000-2003

\section{REFERENCES}

[Ar94] Arkin, E., Held M., Mitchell J., Skiena S., Hamiltionian Triangulations for Fast Rendering. Algorithms-ESA'94, Utrecht, September 1994

[Bh95] Bhattacharya P., Rosenfeld A., Polygonal ribbons in two and three dimensions. Pattern Recognition Vol. 28, No. 5, pp. 769-779, 1995

[Ci00] Peyret F., Jurasz J., Carrel A., Zekri E., Gorham B. The CIRC Project, Revue Générale des Routes no 783, April 2000

[F198] Floriani L., Puppo E., Maillo P., Applications of Computational Geometry to Geographic Information Systems, in J.-R. Sack and J. Urrutia, ed., Handbook of Computational Geometry, Elsevier Science, Amsterdam, 1998

[Os01] Ligier A., Fliedner J., Kajanen J., Peyret F., Open System For Road Information Support. Proc. $18^{\text {th }}$ ISARC, Cracow 2001

[Pe99] Peyret F., The paradigm of computer integrated construction applied to civil-engineering, Proc. $16^{\text {th }}$ ISARC, Madrid 1999

[Pe00] Peyret F., The achievements of the "Computer Integrated Road Construction" project. Proc. $17^{\text {th }}$ ISARC, Taipei 2000

[Ro86] Rosenfeld, A., Axial Representation of Shape. Computer Vision, Graphics and Image Processing (33), No. 2, February 1986, pp. 156-173

[Th01] Thurner H. Quality Assurance and Self Control in Road Construction, Advanced Measurement Technology Proc. $4^{\text {th }}$ International Sympo- sium "Infrastructure Construction Systems and Technologies", Munich, Bauma 2001

[Xi99] Xiang, X., Held, M., and Mitchell, J., Fast and effective stripification of polygonal surface models. In Symposium on Interactive 3D Graphics Proceedings (1999), ACM, pp. 71-78 